\title{
Treatment of Patella Fractures by Compressive External Fixation (CEF)
}

\begin{abstract}
Recent traditional treatment of patella fractures is related with infection and failure of fixation. The complications that we see can be treated surgically which is cost effective and limits the function of the joint. But our locally made CEF device can effectively treat patella fractures with less complications. The transverse fracture of patella with displacement was treated by compressive external fixation, based on Ilizarov tensioned wire construct. All the patients were allowed to do movements in the joints and weight bearing postoperatively.

Eighteen fractures were treated with CEF. 11 patients were treated percutaneously. Sound union was achieved in all the fractures. The CEF device was removed at 6 to 8 weeks. 12 patients regained excellent motion with a mean Insall knee score of 96 points, in an average follow up of 4 years. Minor complications like wire inflammation was observed in 5 patients which was treated by local care and antibiotics. CEF technique is a safe and effective method of treatment in patella fractures with advantages over traditional methods in case with a poor soft tissue envelope. CEF allows rapid recovery and no secondary surgery is needed.
\end{abstract}

Keywords: CEF, Patella fracture
Volume 2 Issue 6 - 2015

\author{
Bari MM, , , ${ }^{2}$ Shahidul Islam, ${ }^{2} \mathrm{NH}$ Shetu, ${ }^{2}$ \\ Mahfuzer Rahman, ${ }^{2}$ Mashiur H Munshi, ${ }^{3}$ Md \\ Golam Mostofa, ${ }^{2}$ Mohammad R Islam Khan ${ }^{3}$ \\ 'Chief Consultant, Bari-llizarov Orthopaedic Centre,Visiting and \\ Honored Prof., Russian Ilizarov Scientific Centre, Russia \\ ${ }^{2}$ Bari-llizarov Orthopaedic Centre, Bangladesh \\ ${ }^{3}$ National Institute of Traumatology and Orthopaedic \\ Rehabilitation, Bangladesh
}

Correspondence: Mofakhkharul Bari, Chief Consultant, BariIlizarov Orthopaedic Centre,Visiting and Honored Professor, Russian Ilizarov Scientific Centre, Kurgan, Tel +88 01819211595 , Email bari.ilizarov3।@gmail.com

Received: May 01, 2015 | Published: June 15, 2015
Abbreviations: CEF, Compressive External Fixation; TBW, Tension Band Wiring

\section{Introduction}

Transverse fracture of patella is the most common pattern, which contributes $70 \%$ of the total 6 . When the fragments are displaced, then surgery is absolutely indicated to maintain the function of the extensor mechanism. However the nature of patella and high tensile forces challenge the standard surgical techniques used for fracture. The traditional method for patella fracture is tension band wiring (TBW) which provides sound union in most cases. ${ }^{2-5}$

Complications like infection upto $12 \%$, fixation failure of $22 \%$ and symptomatic hardware in $20 \%$ to $60 \%$ have been reported. We are reporting here, the Afgan compressive external fixator which was introduced by my friend Mr. M. Ismail Wardak. The technique is based on tensioned biocompatible thin wire external fixation as developed by Academician Prof. Ilizarov for long bones, allows limitless motion. ${ }^{6,7}$ Our aim and purpose of this technique is to review the functional outcomes of patients treated with this novel method. Loss of reduction, and loss of motion, infection and need for second surgery were defined as negative outcomes.

\section{Materials and methods}

From January 2010 to January 2014, 18 patients were treated with displaced fractures of the patella. Displaced fractures means gap of more than $3 \mathrm{~mm}$ and articular steps more than $2 \mathrm{~mm}$. All the 18 patients were treated by CEF. 9 patients were typical two part transverse fractures of the patella.

The following atypical patterns were also treated (Table 1).

Pole fracture too small to permit passage of a wire less than $1 \mathrm{~cm}$ in size, are not indicated for this novel procedure. a. Causes of injures were motor vehicle collision and others. The age ranges were 17 to 65 years (average 35.5 years). Out of 18 patients 15 were men and 3 were women. Time to operation ranged from 8 hours to 10 days. CEF technique is a minimally invasive, percutaneous procedure on 9 closed transverse fractures of patella presenting less than 3 days from the time of injury. The remaining 9 cases were performed through an open procedure. Open reduction was done in all open fractures, upper and lower pole fractures or when accurate reduction was not achieved Percutaneously. All the surgeries were performed under spinal anesthesia. Patient follow up was continued at an average of 4 years postoperatively (range 1, 0 - 4 years).

Table I Atypical Patterns that are Treated

$\begin{array}{cl}\text { Upper pole fracture } & 2 \\ \text { Lower pole fracture } & 3 \\ \text { Stellate fracture } & 2 \\ \text { Three part fracture } & 2\end{array}$

\section{Surgical procedure}

Knee is kept in extension, the fracture fragments are reduced with the help of patellar clamp and it was confirmed by c-arm. Mild traction is applied to the skin medially and laterally to prevent the wires from kinking the skin. Two $1.5 \mathrm{~mm}$ wires are passed horizontally through the proximal fragment. Two additional $1.5 \mathrm{~mm}$ wires are placed in the distal fragment. It is to be noted that the wire pairs be as far from fracture line as possible. This allows for bowing of the wires and compression of the fracture during tensions of the device. The wires are passed through the related holes in the clamp assembly. The clamp assemblies are connected to one another (Figure 1). The wires are secured by the nuts on the clamp assembly. Tensioning of the wires and compression of the fractures are done by distracting the clamps 
from one another by turning the distraction nut until the tension wires are tight. The joint is ranged through flexion and extension while checking the stability of fixation by c-arm.
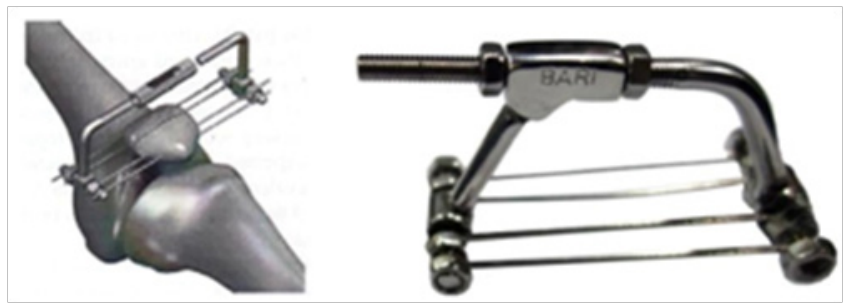

Figure I Compressive External Fixator.

\section{Open reduction technique}

An incision is made vertically over the patella, blood clots are cleaned. The fragments are reduced by clamp. We must palpate the articular surface from side to side to confirm the accurate reduction. The retinaculum is repaired. The wound is closed aseptically. No drains are used. After 1st operative day it is necessary to clean the wire sites with rectified spirit or any kind of antiseptic solution. All the patients were advised to do physical therapy to increase the range of motion. Full range of motion is allowed along with full weight bearing as tolerated by the patient. Sound union was achieved and that was confirmed by x-ray. After 6 to 8 weeks the device was removed.

\section{Results}

Sound union was achieved in all the fragments at an average of 6 to 8 weeks. That was proved clinically and radiologically. Knee flexion was $90^{\circ}$ within 2 to 3 weeks and it was upto $120^{\circ}$ by 4 weeks. The Insall knee score for patient function obtained on 12 patients was 96 points and 2 cases who did not carry our instructions, ultimately resulted in less than $90^{\circ}$ arc of motion. Complications included 3 wire site inflammation which managed by local care. We found articular surface incongruity $(>2 \mathrm{~m})$ because of incomplete primary reduction in 2 cases (Table 2). These two patients with articular incongruity developed patello-femoral osteoarthrosis after 2 years follow up postoperatively (Figure 2).

Table 2 Atypical Patterns that are Treated

\begin{tabular}{clc}
\hline Patients & $\begin{array}{c}\text { Type of } \\
\text { Fractures }\end{array}$ & $\begin{array}{c}\text { Insall Knee } \\
\text { Score }\end{array}$ \\
\hline 9 & Transverse & 96 \\
2 & Upper pole & 90 \\
2 & Comminuted & 92 \\
3 & Lower pole & 95 \\
2 & Stellate & 92
\end{tabular}

Total $=18$
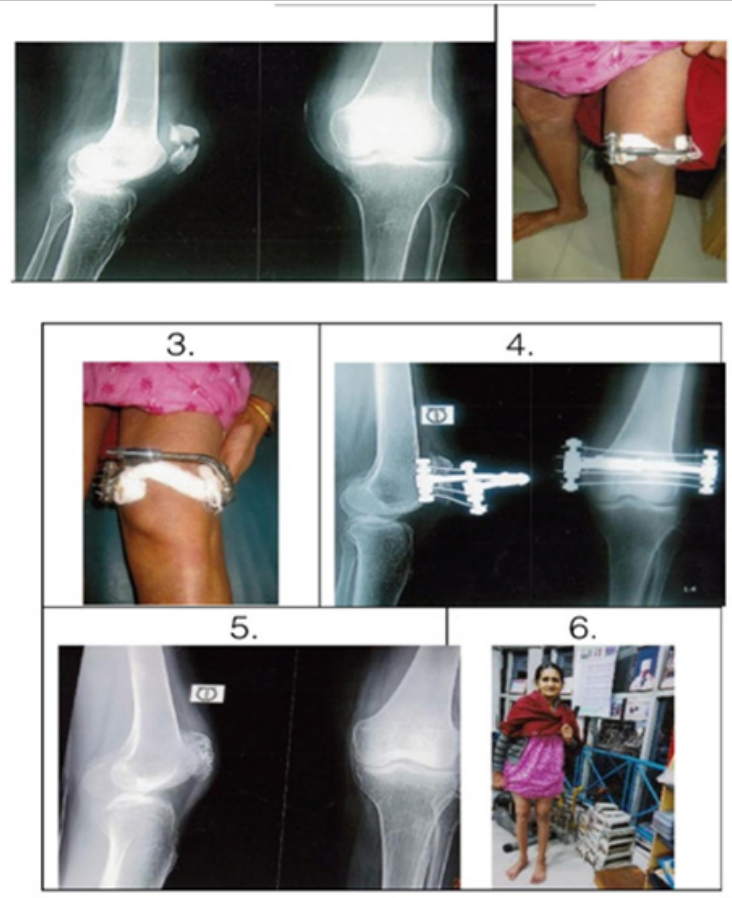

Figure 2

a.Radiograph of transverse fracture of left patella with displacement. b.CEF in the left patella (2nd Post Op).

c.CEF in the left patella follow up after 3 weeks.

d.Radiograph of left patella with CEF in situ (After 3 weeks).

e.Radiographic final result of the patella after removal of CEF

f. Smiling clinical appearance of the patient after treatment.

\section{Discussion}

We are doing traditional modified tension bend wiring since long and it has a great success, ${ }^{3-5}$ but it is associated with some failures with displacement and wire prominence. Our Novel CEF method and device offers advantages compared with traditional method of treatment. All patients healed well without any major complications, despite allowing unrestricted motion. Our cases compares favorably with the recent large series reported by Smith et al., ${ }^{2}$ who analyzed the results of 51 patella fractures treated by TBW. The CEF technique, is an external fixation which allows for adherence to the tenets of articular fracture treatment, accurate reduction with stable fixation for immediate motion, leading to the results we have reported. Early functional activity is an added benefit in our patients which is less cost effective. We did not use any brace. The potential effectiveness of CEF have advantages over the existing other technique. The most significant advantage of CEF is the lack of retained hardware eliminating symptomatic prominence. Infection is not a problem. So in our series we think that we should be considered for all displaced transverse, pole and selected comminuted patella fractures. Open and previously infected fractures are the best candidate for this technique. Yahmis et al. ${ }^{8}$ described the use of ring external fixation to stabilize the comminuted patella fractures. Big advantage is Percutaneously application and in the long term no retained hardware. The technique is a simple, gives absolute stability, provides a significant decrease in 
hospital stay, gives rapid bone healing and rehabilitation and we think that this method should be considered in the treatment of displaced patella fractures, either open or closed.

\section{Acknowledgment}

We are grateful to Dr. Mohammad Ismail Wardak for teaching us this novel technique for treating the patella fractures by CEF.

\section{Disclosure}

The authors report no conflict of interest, financial or otherwise concerning the material or methods used in this study or the findings specified in this paper.

\section{References}

1. Johannes CB, Le TK, Zhou S, et al. The prevalence of chronic pain in United States adults: results of Internet-based survey. J Pain. 2010;11(11):1230-1239.

2. Wardak MI, Siawash AR, Hayda R. Fixation of patella fractures with a minimally invasive tensioned wire method: Compressive external fixation. J Trauma Acute Care Surg. 2012;72(5):1393-1398.

3. Smith ST, Cramer KE, Karges DE, et al. Early complications in the operative treatment of patella fractures. J Orthop Trauma. 1997;11(3):183-187.
4. Schemitsch EH, Weinberg J, McKee, et al. Functional outcome of patella fractures following open reduction and internal fixation. $J$ Orthop Trauma. 1997;13(4):279.

5. Whittle PA, Wood GW. Fractures of lower extremity. In: Terry SC (Ed.), Campbell's operative orthopaedic. (10th edn), Vol. 3, Mosby, Philadelphia, USA, 2003;pp. 2725--872.

6. Weber MJ, Janerki CJ, Leod MCP, et al. Efficacy of various forms of fixation of transverse fractures of the patella. J Bone Joint Surg. 1980;62(2):215-220.

7. Bari MM. A color atlas of limb lengthening, surgical reconstruction and deformity correction by I lizarov technique. 2013;pp. 101.

8. Illizarov GA. Transosseous osteosysthesis, theoretical \& clinical aspects of the regeneration \& growth of tissues, Springer, 1992;pp. 453-495.

9. Yanmis I, Oğuz E, Atesalp AS, et al. Application of circular external fixator under orthopaedic control in comminuted patella fractures: Technique and early results. J Trauma. 2001;60(3):659-663. 